\title{
COMMENSURATE SEQUENCES OF CHARACTERS
}

\author{
A. PELCZYŃSKI
}

(Communicated by William J. Davis)

ABSTRACT. If $\left(a_{j}\right)$ and $\left(b_{j}\right)$ are sequences of characters on compact abelian groups $S$ and $T$ respectively such that for every sequence of scalars $\left(\alpha_{j}\right)$ $\left\|\sum \alpha_{j} a_{j}\right\|_{\infty} \asymp\left\|\sum \alpha_{j} b_{j}\right\|_{\infty}$ then for every $1 \leq p<\infty$ and every sequence $\left(x_{j}\right)$ of elements of an arbitrary Banach space $X$

$$
\int_{S}\left\|\sum x_{j} a_{j}\right\|^{p} d s \asymp \int_{T}\left\|\sum x_{j} b\right\|^{p} d t .
$$

This result generalizes a result of Pisier $\left[\begin{array}{ll}\mathbf{P i} & \mathbf{1}\end{array}\right]$ for Sidon sets. For topological Sidon sets on $\mathbf{R}$ a slightly stronger result holds.

Let $\left(f_{j}\right)$ and $\left(g_{j}\right)$ be sequences of scalar-valued functions in $L^{\infty}(\mu)$ and $L^{\infty}(\nu)$ respectively ( $\mu, \nu$-probability measures). Call $\left(f_{j}\right)$ and $\left(g_{j}\right) d$-commensurate if there exists a $d \geq 1$ such that for every Banach space $X$ and for every $p \in[1, \infty)$ and every sequence $\left(x_{j}\right) \subset X$,

$$
d^{-p} \int\left\|\sum_{j} f_{j} x_{j}\right\|^{p} d \mu \leq \int\left\|\sum_{j} g_{j} x_{j}\right\|^{p} d \nu \leq d^{p} \int\left\|\sum_{j} f_{j} x_{j}\right\|^{p} d \mu .
$$

Pisier [ $\mathbf{P i} \mathbf{1}$ ] observed that if $\left(f_{j}\right)$ and $\left(g_{j}\right)$ are Sidon sets of characters on compact abelian groups then they are commensurate. Using the technique which goes back to Rudin (cf. [R], Theorem 3.1) we generalize Pisier's result to the following.

THEOREM 1. Let $A=\bigcup_{j}\left\{a_{j}\right\}$ and $B=\bigcup_{j}\left\{b_{j}\right\}$ be sets of characters on compact abelian groups $S$ and $T$ respectively. Assume that the correspondence $a_{j} \rightarrow b_{j}$ extends to a linear isomorphism, say $U$ from $C_{A}(S)$ onto $C_{B}(T)$ such that $\|U\|\left\|U^{-1}\right\|=d$.

Then the sequences $\left(a_{j}\right)$ and $\left(b_{j}\right)$ are d-commensurate.

Here by $C_{\Lambda}(G)$ we denote the subspace of continuous complex-valued functions on a compact abelian group $G$ generated by characters from a fixed set $\Lambda$ of the dual group $\widehat{G}$, i.e.

$$
C_{\Lambda}(G)=\left\{f \in C(G): \hat{f}(\gamma)=\int_{G} f(x) \gamma(-x) d x=0 \text { for } \gamma \in \widehat{G} \backslash \Lambda\right\} .
$$

Received by the editors May 28, 1987. Part of this paper has been presented to the American Math. Society in the special session on Functional Analysis at the meeting of AMS in Kent Ohio in the spring of 1987.

1980 Mathematics Subject Classification (1985 Revision). Primary 46E40, 43A46, 43A15.

Key words and phrases. Commensurate sequences, Sidon sets.

This paper was written while the author participated in the Special Year in Modern Analysis at the University of Illinois. The author would like to thank the Mathematics Department of the University for its hospitality and support. 
PrOOF. Fix $t \in T$ and denote by $\delta_{t}$ the point mass at $t$. Let $\phi_{t}^{*}$ be the restriction of $\delta_{t}$ to $C_{B}(T)$. Clearly $\phi_{t}^{*}$ is a linear functional on $C_{B}(T)$ with $\left\|\phi_{t}^{*}\right\| \leq 1$. Obviously for every $b_{j} \in B$,

$$
b_{j}(t)=\phi_{t}^{*}\left(b_{j}\right)=\phi_{t}^{*}\left(U\left(a_{j}\right)\right)=\left(U^{*} \phi_{t}^{*}\right)\left(a_{j}\right) .
$$

Let $\mu_{t}$ be a complex Borel measure on $S$ which via the Riesz Representation Theorem corresonds to the Hahn-Banach extension onto $C(S)$ of the linear functional $U^{*} \phi_{t}^{*}$ on $C_{A}(S)$. Define the measures $\nu_{t}$ and $\bar{\nu}_{t}$ by

$$
\nu_{t}(V)=\mu_{t}(-V) ; \quad \bar{\nu}_{t}(V)=\overline{\mu_{t}(-V)} \quad \text { for Borel } V \subset S .
$$

Then the Fourier coefficients of $\nu_{t}$ at the points of $A$ satisfy

$$
\hat{\nu}_{t}\left(a_{j}\right)=\int_{S} a_{j}(-s) \nu_{t}(d s)=\int_{S} a_{j}(s) \mu_{t}(d s)=b_{j}(t)
$$

clearly the total variation of $\nu_{t}$ satisfies

$$
\left\|\bar{\nu}_{t}\right\|=\left\|\nu_{t}\right\|=\left\|\mu_{t}\right\| \leq\left\|U^{*}\right\|=\|U\| .
$$

Now for a fixed (eventually zero) sequence $\left(x_{j}\right)$ of elements of a Banach space $X$ define the $X$-valued functions $f$ and $f_{t}$ by

$$
f=\sum_{j} x_{j} a_{j} ; \quad f_{t}=\sum_{j} x_{j} b_{j}(t) a_{j} .
$$

Let $f * \nu$ denote the convolution on $S$ of a scalar measure $\nu$ with a vector-valued function $f$, i.e.

$$
(f * \nu)(s)=\int_{S} f(s-\sigma) \nu(d \sigma) \quad \text { for } s \in S .
$$

Then comparing the Fourier coefficients we get

$$
f * \nu_{t}=f_{t} ; \quad f_{t} * \bar{\nu}_{t}=f
$$

Thus, by the vector-valued Young inequality,

$$
\left\|f_{t}\right\|_{p} \leq\|f\|_{p}\left\|\nu_{t}\right\| \leq\|U\|\|f\|_{p} ; \quad\|f\|_{p} \leq\|U\|\left\|f_{t}\right\|_{p}
$$

where $\|f\|_{p}=\left(\int_{S}|f|^{p} d s\right)^{1 / p}$. Integrating these inequalities against the Haar measure $d t$ of $T$ and using the Fubini Theorem (since the sequence $\left(x_{j}\right)$ is eventually zero and the functions $a_{j}$ and $b_{j}$ are continuous, there is no problem of measurability and integrability) we get

$$
\|U\|^{-1}\|f\|_{p} \leq\left(\int_{S} \int_{T}\left\|\sum_{j} a_{j}(s) b_{j}(t) x_{j}\right\|^{p} d s d t\right)^{1 / p} \leq\|U\|\|f\|_{p} .
$$

Exchanging the role of $A$ and $B$ for $g=\sum x_{j} b_{j}$ we get

$$
\left\|U^{-1}\right\|^{-1}\|g\|_{p} \leq\left(\int_{S} \int_{T}\left\|\sum_{j} a_{j}(s) b_{j}(t) x_{j}\right\|^{p} d s d t\right) \leq\left\|U^{-1}\right\|\|g\|_{p}
$$

thus

$$
\left(\|U\|\left\|U^{-1}\right\|\right)^{-1}\|f\|_{p} \leq\|g\|_{p} \leq\|U\|\left\|U^{-1}\right\|\|f\|_{p}
$$


REMARK. Similarly as in Pisier's paper [Pi 1 ] the $L^{p}$-norms in $(1)$ can be replaced by the "averages" $\int_{S} \phi\left\|\sum x_{k} a_{k}\right\| d s$ where $\phi: \mathbf{R}_{+} \rightarrow \mathbf{R}_{+}$is a continuous nondecreasing convex function.

Obviously a sequence of characters which is commensurate with the Rademacher sequence forms a Sidon set. However in that case we have also the following criterion.

PROPOSITION 2. If $\left(a_{k}\right)$ is a sequence of characters on a compact abelian group $S$ such that for some $q$ with $1 \leq q<\infty$ there exists a constant $C$ such that for every finite sequence $\left(x_{k}\right)_{1 \leq k \leq N}(N=1,2, \ldots)$ of elements of every $L^{p}$ space with $2<p \leq \infty$.

$$
\int_{S}\left\|\sum_{k=1}^{N} a_{k}(s) x_{k}\right\|_{L^{p}}^{q} d s \leq C \int_{0}^{1}\left\|\sum_{k=1}^{N} r_{k}(w) x_{k}\right\|_{L^{p}}^{q} d w
$$

then $\bigcup_{k}\left\{a_{k}\right\}$ is a Sidon set.

PrOOF. Fix $p>2$. Specify $L^{p}$ to be $L^{p}(S)$. Fix a sequence of scalars $\left(\alpha_{k}\right)$ and put $x_{k}=\alpha_{k} a_{k}(k=1,2, \ldots)$. Then using the properties of the normalized Haar measure " $d s$ " we get

$$
\begin{aligned}
\int_{S}\left\|\sum_{k=1}^{N} a_{k}(s) \alpha_{k} a_{k}\right\|_{L^{p}(S)}^{q} d s & =\int_{S}\left(\int_{S}\left|\sum_{k=1}^{N} \alpha_{k} a_{k}(s+t)\right|^{p} d t\right)^{q / p} d s \\
& =\int_{S}\left(\int_{S}\left|\sum_{k=1}^{N} \alpha_{k} a_{k}(t)\right|^{p} d t\right)^{q / p} d s \\
& =\left(\int_{S}\left|\sum_{k=1}^{N} \alpha_{k} a_{k}(t)\right|^{p} d t\right)^{q / p} .
\end{aligned}
$$

Observe that for $p>2$,

$$
\left(\int_{S}\left|\sum_{k=1}^{N} \alpha_{k} a_{k}(t)\right|^{p} d t\right)^{1 / p} \geq\left(\sum_{k=1}^{N}\left|\alpha_{k}\right|^{2}\right)^{1 / 2}
$$

On the other hand, by (2), we get

$$
\left(\int_{S}\left|\sum_{k=1}^{N} \alpha_{k} a_{k}(t)\right|^{p} d t\right)^{q / p} \leq C \int_{0}^{1}\left(\int_{S}\left|\sum_{k=1}^{N} \alpha_{k} a_{k}(t) r_{k}(w)\right|^{p} d t\right)^{q / p} d w
$$

(a)

$$
\leq C C_{q}\left(\int_{0}^{1}\left(\int_{S}\left|\sum_{k=1}^{N} \alpha_{k} a_{k}(t) r_{k}(w)\right|^{p} d t\right)^{1 / p} d w\right)^{q}
$$

(b)

$$
\begin{aligned}
& \leq C C_{q} B_{p}^{q}\left(\sum_{k=1}^{N}\left(\int_{S}\left|\alpha_{k} a_{k}(t)\right|^{p}\right)^{2 / p} d t\right)^{q / 2} \\
& =C C_{q} B_{p}^{q}\left(\sum_{k=1}^{N}\left|\alpha_{k}\right|^{2}\right)^{q / 2}
\end{aligned}
$$


where (a) follows from the Kahane-Khinchine inequality, [Kh, p. 20], and (b) follows from the Orlicz inequaliity [O]. Moreover it is well known that the constant $B_{p}$ appearing the Orlicz inequality is dominated by the constant in the Khinchine inequality between $L_{2}$ and $L_{p}$ norms and therefore $B_{p} \leq c \sqrt{p}$ (cf. $[\mathbf{O}$, and $\mathbf{Z}$, Chapter V, $\S 8$, Theorem 8.4] thus we have just shown that there exists a numerical constant $B$ such that

$$
\left(\sum\left|\alpha_{k}\right|^{2}\right)^{1 / 2} \leq\left(\int_{S}\left|\sum \alpha_{k} a_{k}(t)\right|^{p} d t\right)^{1 / p} \leq B \sqrt{p}\left(\sum\left|\alpha_{k}\right|^{2}\right)^{1 / 2}
$$

for all scalars $\left(\alpha_{k}\right)$ and every $p>2$. Hence, by a result of Pisier [Pi 2, or Pi 3], $\bigcup_{k}\left\{\alpha_{k}\right\}$ is a Sidon set.

Our next result concerns topological Sidon sets on the real line $\mathbf{R}$. Recall (cf. $\left[\mathbf{M}\right.$, p. 183] that an increasing sequence of positive real numbers, say $\left(\lambda_{k}\right)$ is called a topological Sidon set if there exist a compact set $K \subset \mathbf{R}$ and $C>0$ such that for every sequence of scalars $\left(\alpha_{k}\right)$

$$
C^{-1} \sum\left|\alpha_{k}\right| \leq \sup _{t \in K}\left|\sum \alpha_{k} e^{i \lambda_{k} t}\right| .
$$

An important example of a topoplogical Sidon set is an increasing sequence $\left(\lambda_{k}^{\prime}\right)$ of positive reals satisfying the Hadamard condition $\inf _{k} \lambda_{k+1} \lambda_{k}^{-1} \geq q>1$ (cf. [M, p. 185].

Our next result shows that if $\left(\lambda_{k}\right)$ is a topological Sidon set then the functions $\left(e^{i \lambda_{k} t}\right)$ considered on any bounded interval $[a, b] \subset R$ are commensurate with the Rademacher functions $\left(r_{k}\right)$.

THEOREM 3. Let $\left(\lambda_{k}\right) \subset \mathbf{R}$ be a topological Sidon set. Then for every interval $[a, b]$ with $-\infty<a<b<+\infty$ there exists a constant $C=C(b-a)$ such that for every $p$ with $1 \leq p<\infty$ and every sequence $\left(x_{j}\right)$ of elements of an arbitrary Banach space $X$

$$
C^{-p} \int_{0}^{1}\left\|\sum r_{k}(w) x_{k}\right\|^{p} d w \leq \int_{a}^{b}\left\|\sum e^{i \lambda_{k} t} x_{k}\right\|^{p} d t \leq C^{p} \int_{0}^{1}\left\|\sum r_{k}(w) x_{k}\right\|^{p} d w .
$$

Proof. Since $\left(\lambda_{k}\right)$ is a topological Sidon set, for every $\delta$ with $b-a>2 \delta>0$ there is a constant $C=C(\delta)$ such that for every scalars $\alpha_{1}, \alpha_{2}, \ldots$

$$
\sum\left|\alpha_{k}\right| \leq C \sup _{|t| \leq \delta}\left|\sum_{k} \alpha_{k} e^{i \lambda_{k} t}\right|
$$

(cf. [DG 1 or M, p. 194, Theorem VII]).

Fix a positive integer $N$. Let $\varepsilon=\left(\varepsilon_{k}\right)_{1 \leq k \leq N}$ be a sequence of \pm 1 of length $N$. It follows from (3) that there exists on the linear span of $\left(e^{i \lambda_{k} t}\right)_{1 \leq k \leq N}$ regarded as a subspace of $C([-\delta, \delta])$ a linear functional of norm $\leq C$ which takes the value $\varepsilon_{k}$ at $e^{i \lambda_{k} t}$ for $k=1,2, \ldots, N$. Thus using the Hahn-Banach and the Riesz Representation Theorems we infer that there is a complex Borel measure $\mu$ on $\mathbf{R}$ such that $\|\mu\| \leq C, \mu$ is concentrated on $[-\delta, \delta], \int_{-\delta}^{\delta} e^{i \lambda_{k} s} \mu(d s)=\varepsilon_{k}$ for $k=1,2, \ldots, N$. Let $|\mu|$ be the unique nonnegative Borel measure on $\mathbf{R}$ such that $\mu$ is absolutely continuous with respect to $|\mu|$ and the Radon-Nikodym derivative $d \mu / d|\mu|$ is a unimodular function $|\mu|$-almost everywhere. 
Now fix a sequence $\left(x_{k}\right)_{1 \leq k \leq N}$ in $X$ and define functions $f: \mathbf{R} \rightarrow X$ and $f_{\varepsilon}: \mathbf{R} \rightarrow$ $X$ by

$$
f(t)=\sum_{k=1}^{N} e^{i \lambda_{k} t} x_{k} ; \quad f_{\varepsilon}(t)=\sum_{k=1}^{N} \varepsilon_{k} e^{i \lambda_{k} t} x_{k} \quad(t \in \mathbf{R}) .
$$

Then for every $t \in \mathbf{R}$

$$
f(t)=\sum_{k=1}^{N} \varepsilon_{k} e^{i \lambda_{k} t} \int_{-\delta}^{\delta} e^{i \lambda_{k} s} \mu(d s)=\int_{-\delta}^{\delta} f_{\varepsilon}(t+s) \mu(d s) .
$$

Hence remembering that $\||\mu|\|=|\mu|([-\delta, \delta]) \leq C$ and using the Hölder inequality we get

$$
\|f(t)\|^{p} \leq C^{p-1} \int_{-\delta}^{\delta}\|f(t+s)\|^{p}|\mu|(d s) \quad(1 \leq p \leq \infty) .
$$

Integrating against the Lebesgue measure and using the Fubini Thoerem we get

$$
\begin{aligned}
\int_{a}^{b}\|f(t)\|^{p} d t & \leq C^{p-1} \int_{a}^{b} \int_{-\delta}^{\delta}\left\|f_{\varepsilon}(t+s)\right\|^{p}|\mu|(d s) d t \\
& =C^{p-1} \int_{-\delta}^{\delta}\left(\int_{a+s}^{b+s}\left\|f_{\varepsilon}(u)\right\|^{p} d u\right)|\mu|(d s) \\
& \leq C^{p-1} \int_{-\delta}^{\delta}\left(\int_{a-\delta}^{b+\delta}\left\|f_{\varepsilon}(u)\right\|^{p} d u\right)|\mu|(d s) \\
& \leq C^{p} \int_{a-\delta}^{b+\delta}\left\|f_{\varepsilon}(u)\right\|^{p} d u .
\end{aligned}
$$

Averaging over all sequences $\varepsilon$ of \pm 1 of length $N$ and using the identity

$$
\underset{\varepsilon}{\operatorname{Average}}\left\|f_{\varepsilon}(u)\right\|^{p}=\int_{0}^{1}\left\|\sum_{k=1}^{N} r_{k}(w) e^{i \lambda_{k} u} x_{k}\right\|^{p} d w
$$

and the Fubini Theorem we obtain

$$
\int_{a}^{b}\|f(t)\|^{p} d t \leq C^{p} \int_{a-\delta}^{b+\delta} \int_{0}^{1}\left\|\sum_{k=1}^{N} r_{k}(w) e^{i \lambda_{k} u} x_{k}\right\|^{p} d w d u .
$$

By the principle of contraction (cf. [Kh, p. 21]) for unimodular complex numbers $\left(e^{i \lambda_{k} u}\right)_{1 \leq k \leq N}$ we get

$$
\int_{0}^{1}\left\|\sum_{k=1}^{N} r_{k}(w) e^{i \lambda_{k} u} x_{k}\right\|^{p} d w \leq(\sqrt{2})^{p} \int_{0}^{1}\left\|\sum_{k=1}^{N} r_{k}(w) x_{k}\right\|^{p} d w .
$$

Thus

$$
\int_{a}^{b}\|f(t)\|^{p} d t \leq(\sqrt{2} C)^{p}(b-a+2 \delta) \int_{0}^{1}\left\|\sum_{k=1}^{N} r_{k}(w) x_{k}\right\|^{p} d w .
$$

To prove the estimate in the opposite direction we replace the interval $[a, b]$ by $[a+\delta, b-\delta]$ and $f$ by $f_{\varepsilon}$. Then we get as in (4)

$$
\int_{a+\delta}^{b-\delta}\left\|f_{\varepsilon}(t)\right\|^{p} d t \leq C^{p} \int_{a}^{b}\|f(u)\|^{p} d u .
$$


Averaging over all sequences of \pm 1 of length $N$ we get

$$
\int_{a+\delta}^{b-\delta} \int_{0}^{1}\left\|\sum_{k=1}^{N} r_{k}(w) e^{i \lambda_{k} t} x_{k}\right\|^{p} d w d t \leq C^{p} \int_{a}^{b}\|f(u)\|^{p} d u .
$$

Next for $t \in[a+\delta, b-\delta]$ put

$$
g_{t}=\prod_{k=1}^{N}\left(1+r_{k} \cos \lambda_{k} t\right)+i \prod_{k=1}^{N}\left(1-r_{k} \sin \lambda_{k} t\right) .
$$

Then $\operatorname{Re} g_{t} \geq 0, \operatorname{Im} g_{t} \geq 0$ and $\int_{0}^{1} \operatorname{Re} g_{t} d w=\int_{0}^{1} \operatorname{Im} g_{t} d w=1$ thus $\int_{0}^{1}\left|g_{t}(w)\right| d w \leq$ 2. Moreover we have

$$
\int_{0}^{1}\left(\sum_{k=1}^{N} r_{k}\left(w+w^{\prime}\right) e^{i \lambda_{k} t} x_{k}\right) g_{t}\left(w^{\prime}\right) d w^{\prime}=\sum_{k=1}^{N} r_{k}(w) e^{i \lambda_{k} t} x_{k}
$$

where $\dot{+}$ denotes the multiplication on the Diadic Group $\{1,-1\}^{\infty}$ transported to $[0,1]$ via the measure preserving map $\left(\varepsilon_{j}\right)_{j \geq 1} \rightarrow \sum_{j=1}^{\infty} 2^{-j-1}\left(1-\varepsilon_{j}\right)$. Thus, by the Young inequality,

$$
\int_{0}^{1}\left\|\sum_{k=1}^{N} r_{k}(w) x_{k}\right\|^{p} d w \leq 2^{p} \int_{0}^{1}\left\|\sum_{k=1}^{N} r_{k}(w) e^{i \lambda_{k} t x_{k}}\right\|^{p} d w .
$$

Thus integrating over $[a+\delta, b-\delta]$ and using (5) we get

$$
(2 C)^{-p}(b-a-2 \delta) \int_{0}^{1}\left\|\sum_{k=1}^{N} r_{k}(w) x_{k}\right\|^{p} d w \leq \int_{a}^{b}\|f(t)\|^{p} d t .
$$

REMARK. Theorem 3 can be generalized to arbitrary topological Sidon sets in locally compact abelian groups studied in [DG 2].

ADKNOWLEDGMENT. The author is indebted to R. Kaufman for supplying the main ingredient in the proof of Theorem 3 and conjecturing Proposition 2. A slightly weaker fact than Theorem 3 has also been proved by O. Blasco.

\section{REFERENCES}

[DG 1] M. Deschamps-Godim, Sur les ensembles de Sidon topologiques, C. R. Acad. Sci. Paris 271 (1970), 1247-1249.

[Dg 2] _ Ensembles de Sidon topologiques, Ann. Inst. Fourier (Grenoble) 22 (1972), 51-79.

[Kh] J.-P. Kahane, Some random series of functions, Cambridge Univ. Press, 1985.

[M] Y. Meyer, Algebraic numbers and harmonic analysis, North-Holland, 1972.

[O] W. Orlicz, Über unbedingte Konvergenz in Funktionräumen. I, II, Studia Math 4 (1933), 33-37, 41-47.

[Pi 1] G. Pisier, Les inégalités de Khinchine-Kahane d'après C. Borel, Séminaire sur le Géométrie des Espaces de Banach 1977-1978, Expose VII, Ecole Polytechnique, Centre de Mathématiques, 1978.

[Pi 2] _ Ensembles de Sidon et processes gaussiens, C. R. Acad. Sci. Paris Sér. A 286 (1978), $671-674$. 
[Pi 3] _ De nouvelles caractérisation des ensembles de Sidon, Math Anal. Appl., Part B, Advances in Math. Suppl. Studies 7 B (1981).

[R] W. Rudin, Trigonometric series with gaps, J. Math. Mech. 9 (1960), 203-227.

[Z] A. Zygmund, Trigonometric series. I, Cambridge Univ. Press, 1959.

Institute of Mathematics, Polish ACAdemy of Sciences, Sniadeckich 8, IP, 00-950 WARSZAWA, POLAND

Department of Mathematics, University of Illinois, 1409 W. Green Street, URBANA, ILLINOIS 61801 